\title{
Article \\ The Lengths of Certain Real Conjugacy Classes and the Related Prime Graph
}

\author{
Siqiang Yang ${ }^{\dagger}$ and Xianhua $\mathrm{Li}^{*}+{ }^{+}$ \\ School of Mathematical Science, Guizhou Normal University, Huaxi District, GuiYang 550000, China; \\ yonstrong@163.com \\ * Correspondence: xhli@suda.edu.cn \\ + These authors contributed equally to this work.
}

check for updates

Citation: Yang, S.; Li, X. The Lengths of Certain Real Conjugacy Classes and the Related Prime Graph. Mathematics 2021, 9, 2060. https:// doi.org/10.3390/math9172060

Academic Editor: Alexander Felshtyn

Received: 22 June 2021

Accepted: 19 August 2021

Published: 26 August 2021

Publisher's Note: MDPI stays neutral with regard to jurisdictional claims in published maps and institutional affiliations.

Copyright: (c) 2021 by the authors. Licensee MDPI, Basel, Switzerland. This article is an open access article distributed under the terms and conditions of the Creative Commons Attribution (CC BY) license (https:// creativecommons.org/licenses/by/ $4.0 /)$.
Abstract: Let $G$ be a finite group. In this paper, we study how certain arithmetical conditions on the conjugacy class lengths of real elements of $G$ influence the structure of $G$. In particular, a new type of prime graph is introduced and studied. We obtain a series of theorems which generalize some existed results.

Keywords: real elements; primary elements; conjugacy class lengths; prime graph; P-groups

\section{Notation and Introduction}

Throughout this paper, the terminology is as follows. All groups are finite and $G$ refers to a group. By a primary element $x$ and a primary $p^{\prime}$-element $y$ for a prime number $p$, we mean that $|x|=r^{n}$ for an arbitrary prime $r$ and $|y|=q^{n}$ for some prime $q \neq p$. Recall that an element $g$ of $G$ is said to be real in $G$ if $g$ and $g^{-1}$ are conjugate in $G$, i.e., there is an $x \in G$ such that $g^{-1}=g^{x}$, and as usual the conjugacy class of the real element $g,\left\{g^{x} \mid x \in G\right\}$, is called a real class of $g$. We denote by $x^{G},\left|x^{G}\right|, \operatorname{Re}(G)$, and $\operatorname{Rp}(G)$ the conjugacy class of $x \in G$, the length of $x^{G}$, the set of real elements of $G$, and the set of real primary elements of $G$, respectively. We denote by $n(\Sigma)$ the number of connected components of a graph $\Sigma$. Define the set $\operatorname{Re}^{*}(G)=\{x \mid x \in \operatorname{Re}(G)$ and $\pi(|x|) \subseteq\{2, p\}$, where $p$ is an arbitrary prime number $\}$. That is, $\operatorname{Re}^{*}(G)$ is the set of real primary elements and real biprimary even order elements of $G$. Let $\operatorname{con}(G), \operatorname{con}_{r}(G)$ and $\operatorname{con}_{r}^{*}(G)$ denote the set of conjugacy classes, the set of real conjugacy classes, the set of conjugacy classes $\left\{g^{G}\right\}$, where $g \in \operatorname{Re}^{*}(G)$, of $G$, respectively. We denote by $\operatorname{cs}(G), \operatorname{cs}_{r}(G)$, and $\operatorname{cs}_{r}^{*}(G)$ the set $\{|C|: C \in \operatorname{con}(G)\},\left\{|C|: C \in \operatorname{con}_{r}(G)\right\}$, and $\left\{|C|: C \in \operatorname{con}_{r}^{*}(G)\right\}$, respectively. Other notation will be explained in due time.

The prime graphs are useful tools for intuitively reflecting the structure of groups, and the investigation of them are still underway. The prime graph related to con $(G)$, denoted by $\Gamma(G)$ (introduced by Alfandary in [1] as $\Gamma^{*}(G)$ ), is a simple graph with vertex set $\mathrm{V}(G)$ the set of primes dividing the length of some $C \in \operatorname{con}(G)$ and there is an edge between two vertices $p$ and $q$ if and only if the product $p q$ divides the length of some $C \in \operatorname{con}(G)$. Prime graphs related to a subset of classes of a group is constructed in the same way. Let $\Gamma_{r}(G)$ be the prime graph related to $\operatorname{con}_{r}(G)$ ( introduced by Dolfi in [2] as $\Gamma^{*}(G)$ ).

The influence of conjugacy class lengths on the structure of groups has been extensively studied in the past two decades (see [3-6]). In particular, the problems of recognizing the solvability of $G$ and the existence of normal (or abelian) $p$-complements of $G$ in this context have been widely investigated, which are also the major themes of our discussion here. Recently, some authors do their research by placing conditions on only real classes of $G$. Fruitful achievements are obtained in this line of study, and it turns out that the lengths of these classes are closely related to some fundamental properties of $G$, see, for instance, $[2,7,8]$. The new techniques developed in those papers are valuable and some analogue methods are employed in this article. 
Now, we mention several notable findings related to real classes. In ([8], Theorem 4.2), Tong-Viet proved that, if the class length of every real primary 2 '-element of $G$ is either a 2-power or not divisible by 4 , then $G$ is solvable. It was shown in ([2], Theorem 6.1) that 2 is not a vertex of $\Gamma_{r}(G)$ if and only if $G$ has a normal Sylow 2-subgrpup $S$ (i.e., $G$ is 2-closed) and $\operatorname{Re}(S) \subseteq \mathrm{Z}(S)$. For odd primes, the outcomes are not that satisfactory. Furthermore, it follows from ([9], Theorem B) that, if the prime graph $\Gamma_{r}(G)$ of $G$ is disconnected, then 2 must be a vertex of $\Gamma_{r}(G)$. These facts confirm repeatedly the importance and the special position of the prime 2 in the study of real classes in groups.

Based on the above observations, in this article, we consider the set $\operatorname{Re}^{*}(G)$ for $G$, which are defined earlier, and introduce the prime graph $\Gamma_{r}^{*}(G)$ related to the classes of elements of $\operatorname{Re}^{*}(G)$, i.e., $\operatorname{con}_{r}^{*}(G)$. Precisely, $\Gamma_{r}^{*}(G)$ is the graph with vertex set $\mathrm{V}_{r}^{*}(G)=$ $\left\{q: q \in \pi(G), q|| C \mid\right.$ for some $\left.C \in \operatorname{con}_{r}^{*}(G)\right\}$, and edge set $E_{r}^{*}(G)=\{\{p, q\}: p \neq q, p q|| C \mid$ for some $\left.C \in \operatorname{con}_{r}^{*}(G)\right\}$.

We pay the majority of attention to class lengths of elements of $\operatorname{Re}^{*}(G)$ in Section 3 and of $\operatorname{Rp}(G)$ in Section 4, respectively, and characterize $G$ of which these lengths have some arithmetical properties. In Section 5, the properties of the prime graph $\Gamma_{r}^{*}(G)$ that we defined and the relation to the structure of $G$ are examined. In this paper, we obtain improvements and variations of some existed results.

\section{Preliminaries}

We will need some results on conjugacy classes, and in particular, on real classes.

Lemma 1. Let $N \unlhd G$. Then, $\left|x^{N}\right||| x^{G} \mid$ for all $x \in N$, and $\left|(x N)^{G / N}\right||| x^{G} \mid$ for all $x N \in G / N$.

Lemma 1 is well known, and we will use it without further reference.

Lemma 2 ([9], Lemma 2.1). (a) If $x \in \operatorname{Re}(G)$ and $\left|x^{G}\right|$ is odd, then $x^{2}=1$.

(b) If $x \in \operatorname{Re}(G)$, then every power of $x$ is a real element of $G$.

(c) If $x \in \operatorname{Re}(G)$, then $x^{t}=x^{-1}$ for some 2-element $t \in G$.

(d) If $N \triangleleft G$ and $|G / N|$ is odd, then $\operatorname{Re}(G)=\operatorname{Re}(N)$.

Lemma 3 ([10], Lemma 2.5). Let $x, y \in G$ be such that $x y=y x$.

(a) Assume $G=C_{G}(x) C_{G}(y)$. Then, $(x y)^{G}=x^{G} \cdot y^{G}$. If in addition $x$ and $y$ are in $\operatorname{Re}(G)$, then $x y \in \operatorname{Re}(G)$.

(b) If $(|x|,|y|)=1$, or if $\langle x\rangle$ and $\langle y\rangle$ are normal in $G$ and have trivial intersection, then $\mathrm{C}_{G}(x y)=\mathrm{C}_{G}(x) \cap \mathrm{C}_{G}(y)$, and therefore $\pi\left(\left|x^{G}\right|\right) \cup \pi\left(\left|y^{G}\right|\right) \subseteq \pi\left(\left|x y^{G}\right|\right)$.

Lemma 4 ([10], Proposition 2.8). Let A be a group of odd order that acts faithfully on a nontrivial elementary abelian 2-group $V$ of order $2^{n}$. Assume that $\mathrm{C}_{V}(A)=1$. Then, the following conditions are equivalent:

(a) For every nontrivial $x \in V,\left|A: \mathrm{C}_{A}(x)\right|$ is a prime number.

(b) There exists a prime $p$ such that, for every nontrivial $x \in V,\left|A: \mathrm{C}_{A}(x)\right|=p$.

Lemma 5 ([2], Proposition 6.4). The following are equivalent:

(a) Every nontrivial element in $\operatorname{Re}(G)$ has even order;

(b) Every element in $\operatorname{Re}(G)$ is a 2-element;

(c) G has a normal Sylow 2-subgroup.

Lemma 6 ([10], Lemma 3.3). Let $x$ be a nontrivial real odd order element of $G$. If $\left|x^{G}\right|$ has prime length $q$, then $q=2$ and $\langle x\rangle \unlhd G$. Furthermore, for every nontrivial $y \in\langle x\rangle$, we have $\mathrm{C}_{G}(y)=\mathrm{C}_{G}(x)$.

The next Lemma gives a neat description of the structure of $G$ when $\operatorname{cs}_{r}(G)=\{1,2, p\}$. 
Lemma 7 ([10], Theorem 3.4). Let $p$ be an odd prime. Then, $\operatorname{cs}_{r}(G)=\{1,2, p\}$ if and only if $G=H \times O$, where $H$ is a dihedral group of order $2 p$ and $\operatorname{cs}_{r}(O)=\{1\}$.

Lemma 8 and 9 are for inductive purposes.

Lemma 8 ([7], Lemma 2.2). Suppose that $N$ is a normal subgroup of $G$ and $x N \in \operatorname{Re}(G / N)$. If $|N|$ or the order of $x N$ in $G / N$ is odd, then $x N=y N$ for some real element $y$ of $G$ (of odd order if the order of $x \mathrm{~N}$ is odd).

Lemma 9. Let $\pi$ be a set of some primes and $N$ a normal subgroup of $G$.

(a) ([11], Lemma 2.3) If $\bar{x}=x N$ is a $\pi$-element, then there exists a $\pi$-element $x_{1}$ of $G$ such that $\bar{x}=\overline{x_{1}}$.

(b) If $|N|$ or the order of $x N$ in $G / N$ is odd, and $\bar{x}=x N$ is a real $\pi$-element, then there exists a real $\pi$-element $x_{1}$ of $G$ such that $\bar{x}=\overline{x_{1}}$.

Proof. (b) By Lemma 8, there exists a real element $y$ of $G$ such that $x N=y N$. Clearly, we can write $y=y_{1} y_{2}$, where $y_{1}$ is a $\pi$-element and $y_{2}$ is a $\pi^{\prime}$-element. As the order of $y N$ is the product of the orders of $y_{1} N$ and $y_{2} N$, we have $y_{2} \in N$; therefore, $y N=y_{1} N$. Since $y_{1}$ is some power of $y$, by Lemma 2(b), $y_{1}$ is real in $G$. Choose $y_{1}=x_{1}$ will meet the conclusion.

Lemma 10 and Lemma 11 are used in Sections 4 and 5, respectively.

Lemma 10. Let $p$ be a prime and $K \unlhd G$ such that $|G|_{p}=|K|_{p}$. Then, $\left|x^{K}\right|_{p}=\left|x^{G}\right|_{p}$ for all non-central $x \in \operatorname{Re}(K)$.

Proof. Let $x \in \operatorname{Re}(K)$ be non-central and let $S_{C} \in \operatorname{Syl}_{p}\left(\mathrm{C}_{G}(x)\right)$ and $S_{G} \in \operatorname{Syl}_{p}(G)$ such that $S_{C} \leq S_{G}$. Since $|G|_{p}=|K|_{p}$, then $S_{C} \leq S_{G} \leq K$. In particular, $S_{G} \in \operatorname{Syl}_{p}(K)$. It follows that $\mathrm{C}_{K}(x)=K \cap \mathrm{C}_{G}(x)$ and $S_{C} \leq K \cap C_{G}(x)$. Therefore, $S_{C} \leq C_{K}(x) \leq C_{G}(x)$ and hence $S_{C}$ is also a Sylow $p$-subgroup of $C_{K}(x)$. Thus, $\left|C_{G}(x)\right|_{p}=\left|S_{C}\right|=\left|C_{K}(x)\right| p$, and then $\left|x^{G}\right|_{p}=\left|G: C_{G}(x)\right|_{p}=\left|S_{G}: S_{C}\right|=\left|K: C_{K}(x)\right|_{p}=\left|x^{K}\right|_{p}$.

Lemma 11. Suppose that $\Gamma_{r}^{*}(G)$ has two connected components with vertex sets $\pi_{1}$ and $\pi_{2}$, where $2 \notin \pi_{2}$. Then, there exists an involution $i \in G$ such that $\left|i^{G}\right|>1$ is a $\pi_{2}$-number and $\mathrm{C}_{G}(i)$ has a normal Sylow 2-subgroup.

Proof. Let $p$ be a prime in $\pi_{2}$. Then, $p$ must divide $\left|i^{G}\right|$ for some $i \in \operatorname{Re}^{*}(G)$. Clearly, every prime divisor of $\left|i^{G}\right|$ is adjacent to $p \in \pi_{2}$; this implies that $\left|i^{G}\right|$ is a nontrivial $\pi_{2}$-number. Hence, $\left|i^{G}\right|>1$ is odd and so $i^{2}=1$ by Lemma 2(a) and thus $i$ is an involution of $G$.

Assume that $\mathrm{C}_{G}(i)$ has a nontrivial real element $x$ of odd order. By Lemma 2(b), we can assume that $|x|=q$, where $q$ is an odd prime. Then, $x i=i x$ and $\left|x^{G}\right|$ is even, so $\left|x^{G}\right|$ is a $\pi_{1}$ number and thus $\left(\left|x^{G}\right|,\left|i^{G}\right|\right)=1$. Lemma 3(a) implies that $x i$ is a real element. Furthermore, since $(|x|,|i|)=1,2 p||(i x)^{G} \mid$ by Lemma 3(b). Since $\pi(|i x|) \subseteq\{2, p\}$, ix $\in \operatorname{Re}^{*}(G)$. Then, $2 \in \pi_{1}$ and $p \in \pi_{2}$ are adjacent in $\Gamma_{r}^{*}(G)$, and we get a contradiction. Therefore, $\mathrm{C}_{G}(i)$ has no nontrivial real element of odd order and thus it has a normal Sylow 2-subgroup by Lemma 5.

We close this section with the remark that trivial class is the only real class of $G$ if and only if $G$ has odd order ([10], Lemma 2.4(iii)), and therefore $G$ has no nontrivial real primary elemnet if and only if $G$ has odd order.

\section{The Lengths of Real Classes}

In ([10], Theorem A), Dolfi proved that $G$ is solvable if all noncentral real classes of $G$ have prime size, and, furthermore, $\operatorname{cs}_{r}(G)$ is contained in a set of the kind $\{1,2\},\{1, p\}$ or 
$\{1,2, p\}$, where $p$ is an odd prime. We show that similar conclusions are true under more general conditions.

We begin with Theorem 1, and a basic result of this section which is the reasoning behind several other results. It shows that the properties of certain real elements can determine those of all the real elements in some ways:

Theorem 1. (1) Every $g \in \operatorname{Re}(G)$ is a 2-element if and only if every $g \in \operatorname{Rp}(G)$ is a 2-element.

(2) Let $p$ be an odd prime, then $\operatorname{cs}_{r}(G)=\{1, p\}$ if and only if $\operatorname{cs}_{r}^{*}(G)=\{1, p\}$.

(3) Let $p$ be an odd prime, then $\operatorname{cs}_{r}(G)=\{1,2, p\}$ if and only if $\operatorname{cs}_{r}^{*}(G)=\{1,2, p\}$.

Proof. (1) Necessity being clear. Assume that every primary real element of $G$ is a 2element, and let $g \in \operatorname{Re}(G)$. We can factor $g$ as $g=g_{1} g_{2} \cdots g_{n}$, where each element $g_{i}$ has distinct prime power orders and commute pairwise. By Lemma 2(b), all $g_{i}$ 's are real in $G$, and therefore, by hypothesis, they are all 2-elements. Thus, $g$ is a 2-element, as required.

(2) First, assume that $\operatorname{cs}_{r}(G)=\{1, p\}$. Let $g \in \operatorname{Re}(G) \backslash \operatorname{Re}^{*}(G)$, then we can write $g=g_{1} g_{2} \cdots g_{n}$ where each element $g_{i}$ have distinct prime power orders and commute pairwise. If $\operatorname{cs}_{r}^{*}(G)=\{1\}$, then all the $g_{i}$ 's are central in $G$, so $g$ is central in $G$ and $\left|g^{G}\right|=1$. We conclude that $\operatorname{cs}_{r}(G)=\{1\}$, a contradiction. If $\operatorname{cs}_{r}^{*}(G)=\{p\}$, then, by Lemma 3(b), it is easy to get $p|| g^{G} \mid$ and so $g \notin Z(G)$. Since $g$ is arbitrary, we get $\operatorname{cs}_{r}(G)=\{p\}$, a contradiction too. Thus, $\mathrm{cs}_{r}^{*}(G)=\{1, p\}$.

Conversely, assume that $\operatorname{cs}_{r}^{*}(G)=\{1, p\}$. For every $g \in \operatorname{Rp}(G)$, we have $\left|g^{G}\right|=1$ or $p$, in each case, by Lemma 2(a), we have $|g|=2$. Now, by (1), we see that every $g \in \operatorname{Re}(G)$ is a 2-element. By assumption $\left|g^{G}\right|=1$ or $p$, and therefore $\mathrm{cs}_{r}(G)=\{1, p\}$, the claim follows.

(3) Assume that $\operatorname{cs}_{r}(G)=\{1,2, p\}$, by Lemma 7 we get $G=H \times O$ where $H$ is a dihedral group of order $2 p$ and $\operatorname{cs}_{r}(O)=\{1\}$. Notice that, for $g=h o \in G=H \times O$, we have $\left|g^{G}\right|=\left|h^{H}\right| \cdot\left|o^{O}\right|$, and $\operatorname{cs}_{r}^{*}(H)=\{1,2, p\}$, and it follows that $\operatorname{cs}_{r}^{*}(G)=\{1,2, p\}$.

Conversely, assume that $\operatorname{cs}_{r}^{*}(G)=\{1,2, p\}$. If no nontrivial primary real element of $G$ has an odd order, then it is easy to see that every real element has an even order. Lemma 5 yields that $G$ has a normal Sylow 2-subgroup $D$, and $\operatorname{Re}(G) \subseteq D$ by Lemma 2(d). That is, $\operatorname{Re}(G) \subseteq \operatorname{Re}^{*}(G)$, and the claim follows. Therefore, we may assume that there exists a nontrivial primary real element $x$ of $G$ having odd order, and clearly $\left|g^{G}\right|=2$.

Let $M=C_{G}(x)$. By Lemma 6 , for every $1 \neq y \in\langle x\rangle, C_{G}(y)=M$ has index 2 in G. Choose element $z \in \operatorname{Re}^{*}(G)$ such that $\left|z^{G}\right|=p$. Observe that $z$ is an involution by Lemma 2(a). Now, if any $1 \neq y \in\langle x\rangle$ centralizes $z$, then, by Lemma 3 , it is easy to see that $y z \in \operatorname{Re}^{*}(G)$ and $\left|(y z)^{G}\right|=2 p$. This is a contradiction by hypothesis. Therefore, $C_{G}(z) \cap$ $\langle x\rangle=1$. In particular, $z \notin M$ and hence $\langle z\rangle$ is a complement of $M$ in $G$. Furthermore, as $\langle x\rangle \cap \mathrm{C}_{M}(z)=1$ and $\left|M: \mathrm{C}_{M}(z)\right||| G: \mathrm{C}_{G}(z) \mid=p$, it follows that $M=\langle x\rangle \times \mathrm{C}_{M}(z)$ and that $|x|=p$. In other words, every nontrivial real primary element of $G$ having an odd order must have order $p$.

Let $g \in \operatorname{Re}(G)$, write $g=g_{1} g_{2} \cdots g_{t}$, where all the $g_{i}{ }^{\prime}$ s are real in $G$ and commute pairwise; in addition, $\left|g_{1}\right|=2^{n_{1}}$ and for $2 \leqslant i \leqslant t,\left|g_{i}\right|=p_{i}^{n_{i}}$ where $p_{i}$ 's are odd primes. By the preceding conclusion, we must have $\left|g_{i}\right|=p$ for $2 \leqslant i \leqslant t$. It follows that $g \in \operatorname{Re}^{*}(G)$, and therefore $\operatorname{Re}(G)=\operatorname{Re}^{*}(G)$. Thus, assertion $\operatorname{cs}_{r}(G)=\{1,2, p\}$ follows.

With Theorem 1 in mind, we found that some existed results can be restated in forms that are a little more general. For instance, considering Lemma 5 and 7, we have the following two corollaries. Corollary 1 describes the groups with all the real primary elements being 2-elements.

Corollary 1. The following are equivalent:

(1) Every element in $\operatorname{Rp}(G)$ is a 2-element.

(2) $G$ has no nontrivial real primary 2'-element.

(3) G has a normal Sylow 2-subgroup. 
Corollary 2 characterizes the structure of $G$ such that the lengths of classes $g^{G}$ for every noncentral $g \in \operatorname{Re}^{*}(G)$ is either 2 or $p$, where $p$ is a fixed odd prime.

Corollary 2. Let $p$ be an odd prime. Then, $\operatorname{cs}_{r}^{*}(G)=\{1,2, p\}$ if and only if $G=H \times O$ where $H$ is a dihedral group of order $2 p$ and $\operatorname{cs}_{r}(O)=\{1\}$.

Theorem 6.1 in [2] states that all real classes of $G$ have odd lengths if and only if $G$ has a normal Sylow 2-subgroup $P$ and $\operatorname{Re}(P) \subseteq \mathrm{Z}(P)$. Here is a similar result.

Theorem 2. Let $P$ be a Sylow 2-subgroup of $G$. Then, all classes of elements of $\operatorname{Rp}(G)$ have odd lengths if and only if $P \triangleleft G$ and $\operatorname{Re}(P) \subseteq \mathrm{Z}(P)$.

Proof. By Theorem 6.1 in [2], we need only show that, if all classes of elements of $\operatorname{Rp}(G)$ have odd lengths, then so do the real classes of $G$. Let the class lengths of all $x \in \operatorname{Rp}(G)$ are odd numbers. We then have $x^{2}=1$ for all $x \in \operatorname{Rp}(G)$, and it is easily seen that all the elements of $\operatorname{Re}(G)$ must be 2-elements. The claim follows by assumption.

The next theorem is another basic result of Section 3, and arguments analogous to those of [10] are used in the proof.

Theorem 3. Assume that $\left|g^{G}\right|$ is either a 2-power or an odd prime for every $g \in \operatorname{Re}^{*}(G)$. Then, $G$ is solvable, and there exists an odd prime $p$ such that $\left|g^{G}\right|$ is either a 2-power or $p$ for every $g \in \operatorname{Re}^{*}(G)$.

Proof. By ([8], Theorem 4.2), we know that $G$ is solvable.

Assume first that $G$ has a normal Sylow 2-subgroup $D$, and set $U=\left\langle g \in \operatorname{Re}(G):\left|g^{G}\right|\right.$ is odd $\rangle$. Observe that, by Lemma $2(a), U$ is an elementary abelian 2-subgroup which is a subgroup of $Z(D)$. Moreover, we have $U \unlhd G$. Let $A=G / C_{G}(U)$. Then, $|A|$ is odd and, by Fitting's Lemma (see ([12], Theorem 4.34), $U=V \times \mathrm{C}_{U}(A)$, where $V=[A, U]$. Note that $A$ acts faithfully on $V$ and that $\mathrm{C}_{V}(A)=\mathrm{C}_{U}(A) \cap V=1$. Every nontrivial $x \in V$ is an involution, so $x \in \operatorname{Re}^{*}(G)$ and $\left|x^{G}\right|=\left|G: C_{G}(x)\right|=\left|A: C_{A}(x)\right|$ is either a power of 2 or an odd prime by hypothesis. However, $2 \nmid\left|x^{G}\right|$ since $x \in \mathrm{Z}(D)$, we have $\left|A: \mathrm{C}_{A}(x)\right|$ is an odd prime. Then, by Lemma 4 , there exists an odd prime $p$ such that $\left|A: \mathrm{C}_{A}(x)\right|=p$ for every nontrivial $x \in V$. Now, if $g \in \operatorname{Re}^{*}(G)$ and $\left|g^{G}\right|$ is an odd prime, then $g=x y$ for suitable elements $x \in V$ and $y \in C_{U}(A)$, and hence $\left|g^{G}\right|=\left|A: C_{A}(x)\right| \in\{1, p\}$. We see that, in this case, the theorem is proved.

We now assume that $G$ does not have a normal Sylow 2-subgroup. Thus, by Lemma 5 , there exists a nontrivial element $x \in \operatorname{Re}(G)$ of odd order. Observe that, by Lemma 2(b), we can assume $|x|=p$, where $p$ is an odd prime. By Lemma 2(a), $\left|x^{G}\right|$ is even, and hence $\left|x^{G}\right|=\left|G: \mathrm{C}_{G}(x)\right|$ is a power of 2 by hypothesis. Now, let $y \in \operatorname{Re}^{*}(G)$ be such that $\left|y^{G}\right|=q$ is an odd prime. Applying again Lemma 2(a), we see that $y$ is an involution.

If we assume $q \neq p$, then $C_{G}(y)$ contains a Sylow $p$-subgroup of $G$; therefore (by a suitable choice of $y$ ), we can assume that $C_{G}(y)$ contains $x$. As $C_{G}(x)$ and $C_{G}(y)$ have a coprime index in $G$, by Lemma 3 we have $x y \in \operatorname{Re}(G)$ and $\left|(x y)^{G}\right|=\mid G: C_{G}(x) \cap$ $\mathrm{C}_{G}(y) \mid=2^{n} q$, where $n$ is a positive integer. Since $\pi(|x y|) \subseteq\{2, p\}$, so $x y \in \operatorname{Re}^{*}(G)$, by hypothesis, this is a contradiction. It follows again that $\left|g^{G}\right|$ is either a power of 2 or $p$ for every $g \in \operatorname{Re}^{*}(G)$.

With minor changes in the preceding proof, we can prove the following corollary.

Corollary 3. Assume that $\left|g^{G}\right|$ is a prime for every $g \in \operatorname{Re}^{*}(G)$. Then, $G$ is solvable, and there exists a prime $p$ such that $\operatorname{cs}_{r}^{*}(G) \subseteq\{1,2, p\}$.

\section{The Class Lengths of Primary Real Elements}

Recognizing $p$-nilpotency (i.e., has a normal $p$-complement or not) is clearly an important problem. In this section, we provide some evidence for a conjecture proposed in [8], 
which said that, if the lengths of all the nontrivial real classes in $G$ have the same 2-part, then $\mathrm{O}^{2^{\prime}}(G)$ is 2-nilpotent; in particular, $G$ is solvable. In Theorem 4, we prove that, with only real classes of primary elements being considered, this conjecture is held under some condition on the Sylow 2-subgroups.

In ([13], Theorem 3.4), Navarro et al. showed that all real class lengths of $G$ are powers of 2 if and only if $G$ has a normal 2-complement $K$ and $\operatorname{Re}(G) \subseteq \mathrm{C}_{G}(K)$. However, when $p$ is an arbitrary prime, the situation for the existence of a normal $p$-complement is different. We also remark that, in ([14], Lemma 2.2), Chen and Zhao proved that, if $\left|x^{G}\right|$ is a $p$-number for every primary $p^{\prime}$-element $x$ of $G$, then $G$ is a solvable group with abelian $p$-complements. Here, we obtain Theorem 5 as an analogous result.

At the end of this section, a particular class of groups is concerned, namely, the $\mathbf{P}$ groups. For every subgroup $H$ of $G$, if $H<P_{G}(H)$ where $P_{G}(H)=\langle x| x \in G,\langle x\rangle H=$ $H\langle x\rangle\rangle$, then $G$ is said to satisfy the permutizer condition $\mathbf{P}$, or to be a $\mathbf{P}$-group. In [15], the authors proved that $\mathbf{P}$-groups are solvable by making use of the Classification Theorem of Finite Simple Groups (CFSG), they also proved that every maximal subgroup of a $\mathbf{P}$ group has index 4 or a prime. It is meaningful to consider how close such groups come to being supersolvable.

Theorem 4. Suppose that $\left|x^{G}\right|_{2}=2^{n}$ for all non-central $x \in \operatorname{Rp}(G)$. Assume further that $G$ has a Sylow 2-subgroup $S$ with $\operatorname{Re}(S) \subseteq \mathrm{Z}(S)$. Then, $G$ is solvable and $\mathrm{O}^{2}(G)$ is 2-nilpotent.

Proof. We first prove that $G$ is solvable. Let $G$ be a counterexample of minimal order. Then, $G$ is non-solvable and has no normal Sylow 2-subgroup. By Corollary 1, there exists a nontrivial real primary $2^{\prime}$-element $z \in G$. Clearly, $z \notin Z(G)$ and so $\left|z^{G}\right|$ is even. Thus, $\left|z^{G}\right|_{2}=2^{n} \geqslant 2$. That is, $\left|x^{G}\right|_{2}=2^{n} \geqslant 2$ for all non-central $x \in \operatorname{Rp}(G)$.

Now we claim that every nontrivial real 2-element of $G / \mathrm{O}_{2^{\prime}}(G)$ lies in the center of $G / \mathrm{O}_{2^{\prime}}(G)$. Let $x \mathrm{O}_{2^{\prime}}(G)$ be a nontrivial real 2-element of $G / \mathrm{O}_{2^{\prime}}(G)$. By Lemma $9(\mathrm{~b})$, there exists a real 2-element $y$ of $G$ such that $x \mathrm{O}_{2^{\prime}}(G)=y \mathrm{O}_{2^{\prime}}(G)$. Considering $y$, we have $y^{t}=y^{-1}$ for some 2-element $t \in G$ by Lemma 2(c). Write $U=\langle y, t\rangle$. Since $t$ normalizes $\langle y\rangle, U$ is a 2-subgroup of $G$. We have $U^{g} \leq S$ for some $g \in G$ by the Sylow theorem. We want to show that $y \in U$ is a central involution of $G$. Notice that, if $y^{g}$ is a central involution of $G$, then so is $y$. Therefore, we may assume that $U \leq S$. Since $y \in \operatorname{Re}^{*}(U)$, then $y \in \operatorname{Re}(S) \subseteq Z(S)$, so it follows easily that $y$ is an involution and $\left|y^{G}\right|$ is odd. However, by the first paragraph, $\left|y^{G}\right|$ is even if $y$ is not central, and this forces $y \in Z(G)$. We conclude that $y$ is a central involution of $G$, hence $x \mathrm{O}_{2^{\prime}}(G)=y \mathrm{O}_{2^{\prime}}(G)$ is also a central involution of $\mathrm{G} / \mathrm{O}_{2^{\prime}}(G)$, and the claim follows.

Since $\mathrm{O}_{2^{\prime}}\left(G / \mathrm{O}_{2^{\prime}}(G)\right)=1$, by ([16], the main theorem), the last term of the derived series of $G / \mathrm{O}_{2^{\prime}}(G)$ is isomorphic to $G_{1} \times G_{2} \times \cdots \times G_{S}$, where each $G_{i}$ is isomorphic to either $\mathrm{SL}_{2}(q)$ with odd number $q>5$, or $2 \cdot \mathrm{A}_{7}$, the perfect double cover of $\mathrm{A}_{7}$. We claim that, for some $i$, there exists a real element $a$ of $G_{i}$, and therefore of $G / \mathrm{O}_{2^{\prime}}(G)$, such that $|a|=2^{m} \geqslant 4$. First, assume that $G_{i} \cong 2 \cdot A_{7}$ for some $i$. Using [17], $G_{i}$ has a real element of order 4. Next, assume that $G_{i} \cong \mathrm{SL}_{2}(q)$ with odd number $q>5$. As we know (see ([18], Theorem 2.8.3)), the Sylow 2-subgroup $S$ of $\mathrm{SL}_{2}(q)$ is a generalized quaternion group $Q_{2^{k+1}}$ of order $2^{k+1} \geqslant 8$. From its presentation $\left\langle a, b: a^{2^{k-1}}=b^{2}, a^{2^{k}}=1, b^{-1} a b=a^{-1}\right\rangle$, we obtain a real element $a$ of $\mathrm{SL}_{2}(q)$ of order $2^{k} \geqslant 4$. Of course, $a$ is also real in $G / \mathrm{O}_{2^{\prime}}(G)$ as $G_{i}$ is a subgroup of $G / \mathrm{O}_{2^{\prime}}(G)$. The claim follows. a must lie in the center of $G / \mathrm{O}_{2^{\prime}}(G)$, which yields $a \in \mathrm{Z}\left(G_{i}\right)$. Notice that $\mid \mathrm{Z}\left(G_{i} \mid=2\right.$ for all $i \geqslant 1$, and we get a contradiction. Therefore, $G$ is solvable.

Suppose that $G>\mathrm{O}^{2^{\prime}}(G)$. Using Lemma 10 where we let $p=2$ and $K=\mathrm{O}^{2^{\prime}}(G)$, we see that $\left|x^{K}\right|_{2}=2^{n}$ for all non-central $x \in \operatorname{Rp}(K)$ and by induction $\mathrm{O}^{2^{\prime}}(G)$ is 2-nilpotent. Hence, we may assume that $G=\mathrm{O}^{2^{\prime}}(G)$. Let $\bar{G}=G / \mathrm{O}_{2^{\prime}}(G)$ and use bar as the customary. Let $\bar{P}=\mathrm{O}_{2}(\bar{G}) \cdot \bar{G}$ has a Hall 2'-subgroup $\bar{H}$ since it is solvable. By the second paragraph, we see that every nontrivial real 2-element of $\bar{G}$ lies in $Z(\bar{G})$. This means that the conjugation action of $\bar{H}$ on $\bar{P}$ fixes all real elements of order 2 or 4 , and then $\bar{H}$ centralizes $\bar{P}$ by ([19], 
Theorem B). By ([12], Theorem 3.21), we get $\bar{H} \leq \mathrm{C}_{\bar{G}}(\bar{P}) \leq \bar{P}$, which yields $\bar{H}=1$. Hence, $\bar{P}=\bar{G}$ is a 2-group and we conclude that $G$ is 2-nilpotent.

Theorem 5. Let $p$ be a prime, $\left|x^{G}\right|$ a power of $p$ for every 2-element, and every p-element $x$ of $G$, and also for every real primary $p^{\prime}$-element $x$ of $G$. Then, $G$ has a normal p-complement.

Proof. First, by ([8], Theorem 4.2), we see that $G$ is a solvable group. Next, we show that $G$ has a normal $p$-complement by induction on $|G|$. Let $P \in \operatorname{Syl}_{p}(G)$ and $M$ be a normal maximal subgroup of $G$. Then, $|G / M|=q$ a prime. Note that $\operatorname{Rp}(M) \subseteq \operatorname{Rp}(G)$ and $\left|x^{M}\right||| x^{G} \mid$ for all $x \in M$, By induction, we know that $M$ has a normal $p$-complement $H$. Hence, we may assume that $q \neq p$.

Let $\bar{G}=G / H$. We claim that $\bar{G}$ inherits the hypothesis on $G$. Let $\bar{x}$ be a $p$-element (2-element, resp. ) of $\bar{G}$. By Lemma 9(a), there exists a $p$-element (2-element, resp. ) $x_{1}$ of $G$ such that $\bar{x}=\overline{x_{1}}$. Therefore, $\left|\bar{x}^{\bar{G}}\right|=\left|\bar{x}_{1} \bar{G}\right||| x_{1}^{G} \mid$, which yields that $\left|\bar{x}^{\bar{G}}\right|$ is a $p$-power. Let $\bar{y}$ be a real primary $p^{\prime}$-element of $\bar{G}$, and we can assume that $|\bar{y}|=r^{m}$ for some prime $r \neq 2$, since otherwise, by hypothesis and Lemma 9 (a), we easily get that $\left|\bar{y}^{\bar{G}}\right|$ is a power of $p$. By Lemma $9(\mathbf{b})$, there exists a real $r$-element $y_{1}$ of $G$ such that $\bar{y}=\overline{y_{1}}$. Hence, $|\bar{y} \bar{G}|=\left|\overline{y_{1}} \bar{G}\right|\left|y_{1}^{G}\right|$; again, $\left|\bar{y}^{\bar{G}}\right|$ is also a power of $p$. The claim follows. Now, if $H>1$, then $G / H$ has a normal $p$-complement by induction, and therefore $G$ has a normal $p$-complement.

Based on the above, we may assume that $H=1$. In particular, $M=P \triangleleft G$. Now, let $K$ be a Sylow $q$-subgroup of $G$. We have that $K$ acts coprimely on $P$. We claim that every element of $P$ is $K$-invariant. Let $x \in P$. Then, $\left|G: \mathrm{C}_{G}(x)\right|$ is a $p$-power since $x$ is a $p$-element of $G$. Therefore, there exists $y \in P$ such that $K^{y^{-1}} \subseteq \mathrm{C}_{G}(x)$. Then, $K \subseteq \mathrm{C}_{G}\left(x^{y}\right)$, and we deduce that the conjugacy class $x^{P}=\left(x^{y}\right)^{P}$ is $K$-invariant. It follows that the action of $K$ on $P$ induces an action of $K$ on the classes in $P$, and, for every class $C$ in $P, K$ fixes some element of $C$, say $x_{C}$. Set $J=\left\langle x_{C} \mid C \in \operatorname{con}(P)\right\rangle$. Clearly, $K$ acts trivially on $J$. Since $J$ contains an element from each class in $P$, we get $P=\bigcup_{v \in P} J^{v}$. Hence, $P=J$ and the action of $K$ on $P$ is trivial, which implies that $G$ has a normal $p$-complement $K$. This completes the proof.

Remark 1. When $p=2$, Theorem 5 still holds of course.

By the inductive method and utilizing the properties of $\mathbf{P}$-groups mentioned before, we give a sufficient condition for a P-group to be supersolvable. It seems that to date that there is no similar result of this kind.

Theorem 6. Let $G$ be a $\boldsymbol{P}$-group. Suppose that $8 \nmid\left|x^{G}\right|$ for every 2-element and every real primary element $x \in G$. Then, $G$ is supersolvable.

Proof. Assume that the theorem is false and let $G$ be a counterexample. Thus, $G$ is not supersolvable.

If every maximal subgroup of $G$ has index a prime, then $G$ is supersolvable. Hence, $G$ has a maximal subgroup $M$ such that $|G: M|=4$. Therefore, $|G|$ is even. Since $G$ is a Pgroup, $G$ is solvable by the preceding remarks. There exists a minimal normal subgroup $N$ of $G$ such that $N$ is elementary abelian of order $p^{n}$ where $p$ is a prime. Consider $\bar{G}=G / N$. It can be shown that $\bar{G}$ is a P-group. We may suppose that $n>1$, since, otherwise, $G$ is supersolvable as required. Let $\bar{x}$ be a real primary $2^{\prime}$-element of $\bar{G}$, by Lemma $9(\mathrm{~b})$, there exists a real primary $2^{\prime}$-element $x_{1}$ of $G$ such that $\bar{x}=\overline{x_{1}}$. We see that $\left|\bar{x}^{\bar{G}}\right|=\left|\bar{x}_{1} \bar{G}\right||| x_{1}^{G} \mid$, which yields $8 \nmid\left|\bar{x}^{\bar{G}}\right|$. On the other hand, let $\bar{y}$ be a 2-element of $\bar{G}$; by Lemma $9(a)$, it is easy to deduce that $8 \nmid\left|\bar{y}^{\bar{G}}\right|$. This shows that $\bar{G}$ inherits our hypothesis and thus is supersolvable. Note that the class of finite supersolvable groups is a saturated formation, hence $\Phi(G)=1$ and $N$ is the unique minimal normal subgroup of $G$. From these facts, we obtain a maximal 
subgroup $M$ of $G$ such that $G=M N$ and $M \cap N=1$. Since $|G: M|$ is a prime or 4, we must have $|N|=4$, that is, $p=n=2$.

Consider the permutation representation of $G$ on $M$. We see that $G$ is isomorphic to a subgroup of $S_{4}$. Hence, $G \cong \mathrm{S}_{4}$ or $G \cong \mathrm{A}_{4}$ since $G$ is not supersolvable. Furthermore, it is easy to check that $\mathrm{A}_{4}$ is not a P-group, so $G \cong \mathrm{S}_{4}$. We remark that every element of $\mathrm{S}_{4}$ is real. Now, for an element $x$ of order 3 , for example, permutation (123), since $\left|C_{G}(x)\right|=3$, we have $\left|x^{G}\right|=\left|G: C_{G}(x)\right|=8$, a contradiction to our hypothesis. Therefore, we conclude that $G$ is supersolvable.

Remark 2. By Lemma 9(b), for real 2-elememts, the inductive method may not work out when applied to quotient groups; therefore, assumptions for (not real) 2-elements are needed in Theorems 5 and 6.

\section{The Prime Graph}

The implications of the class lengths of $G$ on the structure of $G$ can be studied via prime graphs. Interestingly, prime graphs related to different subsets of classes exhibit some similarities in their graphic features. For instance, it was shown that $n(\Gamma(G)) \leqslant 2$ and $n\left(\Gamma_{r}(G)\right) \leqslant 2$ for any group $G$ (see $\left.[1,2]\right)$. In addition, $\Gamma_{p}^{\prime}(G) \leqslant 2$ if $G$ is a $p$-solvable group, where $\Gamma_{p}^{\prime}(G)$ is the prime graph related to the set of classes of $p^{\prime}$-elements of $G$ (see [20]).

By the proof of Theorem 2, the condition that all the elements of $\operatorname{Rp}(G)$ have odd number class lengths is equivalent to saying that all the elements of $\operatorname{Re}(G)$, and therefore of $\operatorname{Re}^{*}(G)$, have odd number class lengths, hence Theorem 2 shows that 2 is not a vertex of $\Gamma_{r}^{*}(G)$ if and only if $G$ has a normal Sylow 2-subgroup $P$ and $\operatorname{Re}(P) \subseteq \mathrm{Z}(P)$.

Our Theorem 7 determines the number of connected components of the prime graphs $\Gamma_{r}^{*}(G)$, and the finding is consistent with those regarding $\Gamma(G)$ and $\Gamma_{r}(G)$. For convenience, let $g \in \operatorname{Re}^{*}(G)$ be a non-central element, and we denote by $\Delta_{G}(g)$ the connected component of $\Gamma_{r}^{*}(G)$ that contains the set of vertices $\pi\left(\left|g^{G}\right|\right)$, and $\Delta_{G}(q)$ the connected component of $\Gamma_{r}^{*}(G)$ which $q$ belongs to, where $q$ is a prime.

Considering $\Gamma_{r}(G)$, by ([9], Theorem 3.4), we have that $G$ is solvable if $\Gamma_{r}(G)$ is disconnected. It is tempting to wonder wether the corresponding conclusion for $\Gamma_{r}^{*}(G)$ is valid or not. However, we are unable to prove or disprove this yet. Nevertheless, for a very restrictive case, we have Theorem 9, the proof of which is via a series of lemmas and theorems, in particular, Theorem 7 and 8 are employed.

Theorem 7. For any group $G, n\left(\Gamma_{r}^{*}(G)\right) \leqslant 2$.

Proof. Working by contradiction, let $G$ be a group of minimal order such that $n\left(\Gamma_{r}^{*}(G) \geqslant 3\right.$. Assume first that there exists a nontrivial element of odd order $x \in \operatorname{Re}^{*}(G)$. By taking a suitable power, we can assume that $|x|=p$ with $p$ an odd prime. By Lemma 2(a), we have $\Delta_{G}(x)=\Delta_{G}(2)$. Since $n\left(\Gamma_{r}^{*}(G)\right) \geqslant 3$, there is a connected component $\Delta$ of $\Gamma_{r}^{*}(G)$ with $\Delta \neq \Delta_{G}(x)$ and $\Delta \neq \Delta_{G}(p)$. Choose $y \in \operatorname{Re}^{*}(G)$ such that $\Delta=\Delta_{G}(y)$. Because $2 \notin \Delta, y$ is an involution. Furthermore, as $p \notin \Delta, y$ commutes with a Sylow $p$-subgroup of $G$ and, up to conjugation, we can assume that $x$ and $y$ commute. Note that $x y \in \operatorname{Re}^{*}(G)$, so, by Lemma 3, we get the contradiction $\Delta_{G}(x)=\Delta_{G}(x y)=\Delta_{G}(y)$.

We see that all elements of $\operatorname{Re}^{*}(G)$ are 2-elements, and so are all elements of $\operatorname{Re}(G)$. Hence, by Lemma 5, $G$ has a normal Sylow 2-subgroup $P$. In particular, $G$ is solvable by the Feit-Thompson theorem. Let $N$ be a maximal normal subgroup of $G$ containing $P$. Then, $[G: N]=q$ an odd prime and $\operatorname{Re}(G)=\operatorname{Re}(N)$ by Lemma 2(d). If $x \in \operatorname{Re}^{*}(G)$, then $\pi\left(\left|x^{N}\right|\right) \subseteq \pi\left(\left|x^{G}\right|\right) \subseteq \pi\left(\left|x^{N}\right|\right) \cup\{q\}$. By minimality, $n\left(\Gamma_{r}^{*}(N)\right) \leqslant 2$, and this implies that a connected component of $\Gamma_{r}^{*}(G)$ must consist of the single prime $q$, and $q \notin \mathrm{V}_{r}^{*}(N)$. Thus, if $g \in \operatorname{Re}^{*}(G)$ and $q \in \pi\left(\left|g^{G}\right|\right)$, then $\left|g^{G}\right|$ is a $q$-power and $g \in Z(N)$. In addition, by Lemma $2(\mathrm{a}), g$ is an involution.

Let $Z=Z(P) \triangleleft G$. Since $P$ lies in the kernel of the action of $N$ on $Z$, we have the decomposition $Z=C_{Z}(N) \times[Z, N]$, with $C_{Z}(N),[Z, N] \triangleleft G$. Let $x \in \operatorname{Re}^{*}(G)$ with $\Delta_{G}(x)=\{q\}$. Then, $x \in Z \cap C_{G}(N)=C_{Z}(N)$. Observe that $[Z, N] \neq 1$, as otherwise every real class of elements of $\operatorname{Re}^{*}(G)$ that has an odd length would have $q$-power length 
and hence $n\left(\Gamma_{r}^{*}(G)\right) \leqslant 2$. Let $y \in[Z, N]$ be an involution. Then, from $N \subseteq \mathrm{C}_{G}(x)$ and $N \nsubseteq \mathrm{C}_{G}(y)$, we obtain $\left(\left|x^{G}\right|,\left|y^{G}\right|\right)=1$, so, by Lemma 3(a), it follows $x y \in \operatorname{Re}(G)$. Of course, $x y \in \operatorname{Re}^{*}(G)$. Since $x$ and $y$ lie in normal subgroups of $G$ which intersect trivially, we get $\mathrm{C}_{G}(x y)=\mathrm{C}_{G}(x) \cap \mathrm{C}_{G}(y)$ and therefore $\pi\left(\left|(x y)^{G}\right|\right) \supseteq \pi\left(\left|x^{G}\right|\right) \cap \pi\left(\left|y^{G}\right|\right)$. This gives $\Delta_{G}(x)=\Delta_{G}(x y)=\Delta_{G}(y)$, a contradiction.

If $N \unlhd G$, by the fact that $\operatorname{Re}^{*}(N) \subseteq \operatorname{Re}^{*}(G)$, we easily see that $\Gamma_{r}^{*}(N)$ is a subgraph of $\Gamma_{r}^{*}(G)$. However, it may not be true that $\Gamma_{r}^{*}(G / N)$ is a subgraph of $\Gamma_{r}^{*}(G)$. However, this is the case if $|N|$ is odd.

Lemma 12. Let $N \unlhd G$ with $|N|$ odd. Then, $\Gamma_{r}^{*}(G / N)$ is a subgraph of $\Gamma_{r}^{*}(G)$.

Proof. First, we argue that $\mathrm{V}_{r}^{*}(G / N) \subseteq \mathrm{V}_{r}^{*}(G)$. Let $p \in \mathrm{V}_{r}^{*}(G / N)$ and let $x N \in \operatorname{Re}^{*}(G / N)$ such that $p||(x N)^{G / N} \mid$. By Lemma 9(b), there exists an element $y \in \operatorname{Re}^{*}(G)$ such that $x N=y N$. Since $\left|(x N)^{G / N}\right|=\left|(y N)^{G / N}\right||| y^{G}|, p|\left|y^{G}\right|$ and $p \in \mathrm{V}_{r}^{*}(G)$.

With a similar argument, we can prove that, if there are two different vertices $p, q \in$ $\mathrm{V}_{r}^{*}(G / N)$, adjacent in $\Gamma_{r}^{*}(G / N)$, then $p, q$ are adjacent in $\Gamma_{r}^{*}(G)$ by using Lemma $9(\mathrm{~b})$ again. Thus, $\Gamma_{r}^{*}(G / N)$ is a subgraph of $\Gamma_{r}^{*}(G)$.

Let $H$ be a subgroup or a quotient group of $G$. Assume that $\Gamma_{r}^{*}(H)$ is a subgraph of $\Gamma_{r}^{*}(G)$, and $\Gamma_{r}^{*}(G)$ is also disconnected having two connected components with vertex sets $\pi_{1}$ and $\pi_{2}$, respectively. To show that $\Gamma_{r}^{*}(H)$ is disconnected, it suffices to show that $\mathrm{V}_{r}^{*}(H) \cap \pi_{i} \neq \varnothing$ for $i=1,2$, or, equivalently, there exists two elements $u_{1}, u_{2}$ of $\operatorname{Re}^{*}(H)$ such that $\pi\left(\left|u_{i}^{H}\right|\right) \cap \pi_{i} \neq \varnothing$ for $i=1,2$.

Theorem 8. Suppose that, for a group $G, \Gamma_{r}^{*}(G)$ has two connected components with vertex sets $\pi_{1}$ and $\pi_{2}$, where $2 \notin \pi_{2}$. Then:

(1) If $G$ is not 2-closed and $M \unlhd G$ with $|G: M|$ odd, then $\Gamma_{r}^{*}(M)$ is disconnected.

(2) If $N \unlhd G$ with $|N|$ odd and assuming further that $G=\mathrm{O}^{2^{\prime}}(G)$ is not 2-nilpotent, then $\Gamma_{r}^{*}(G / N)$ is disconnected.

Proof. By Lemma $11, G$ has an involution $i$ such that $\left|i^{G}\right|>1$ is a $\pi_{2}$-number and $\mathrm{C}_{G}(i)$ has a normal Sylow 2-subgroup $S$.

For (1), let $M \unlhd G$ with $|G: M|$ being odd. We have $V_{r}^{*}(M) \subseteq V_{r}^{*}(G)=\pi_{1} \cup \pi_{1}$. Notice that, if $M$ is 2-closed, then $G$ is also 2-closed, hence we may assume that $M$ is not 2-closed. By Theorem 2, 2 divides the class length of some element of $\operatorname{Rp}(M)$ and hence $2 \in \mathrm{V}_{r}^{*}(M) \cap \pi_{1}$. Now, $M$ contains every real element of $G$ by Lemma $2(\mathrm{~d})$; then, $i \in M$. If $M \leq \mathrm{C}_{G}(i)$, then $S \unlhd M$ as $|G: M|$ is odd, so $M$ is 2 -closed, a contradiction. Thus, $\left|i^{M}\right|>1$ and then $V_{r}^{*}(M) \cap \pi_{2} \neq \varnothing$. As $\Gamma_{r}^{*}(M)$ is a subgraph of $\Gamma_{r}^{*}(G)$ and $V_{r}^{*}(M) \cap \pi_{i} \neq \varnothing$ for each $i=1,2$, we conclude that $\Gamma_{r}^{*}(M)$ is disconnected.

For (2), let $N \unlhd G$ with $|N|$ being odd. By Lemma $12, \Gamma_{r}^{*}(G / N)$ is a subgraph of $\Gamma_{r}^{*}(G)$. In particular, $\mathrm{V}_{r}^{*}(G / N) \subseteq \mathrm{V}_{r}^{*}(G)=\pi_{1} \cup \pi_{1}$. If $G / N$ is 2-closed, then $S N \unlhd G$ is of an odd index, thus $G=S N$ since $G=\mathrm{O}^{2^{\prime}}(G)$. In other words, $G$ is 2-nilpotent, by hypothesis, this is a contradiction. Hence, we may assume that $G / N$ is not 2-closed.

The element $i N \in G / N$ is clearly an involution. If $i N \in Z(G / N)$, we have $G / N=$ $\mathrm{C}_{G / N}(i N)=\mathrm{C}_{G}(i) N / N$, where the latter equality follows from ([12], Lemma 7.7). Hence, $S N / N$ is a normal Sylow 2-subgroup of $G / N$, a contradiction. Therefore, $i N \notin Z(G / N)$ and $\left|(i N)^{G / N}\right|>1$, so $\mathrm{V}_{r}^{*}(G / N) \cap \pi_{2} \neq \varnothing$. On the other hand, as $G / N$ is not 2-closed, by Theorem 2, again $2 \in \mathrm{V}_{r}^{*}(G / N) \cap \pi_{1}$. We conclude that $\Gamma_{r}^{*}(G / N)$ is disconnected.

Finally, recall that the famous Feit-Thompson Theorem from 1963 states that every group of odd order is solvable. Therefore, we know that $G$ is solvable if $|G|=2 r$ where $r$ is a prime by ([12], Theorem 1.35). This conclusion as well as some arguments similar to those in the proof of ([9], Theorem 3.4) are applied to get the following theorem. 
Theorem 9. Let $|G|=2^{n} r$, where $n \leqslant 2$ and $r$ is an odd number; if $\Gamma_{r}^{*}(G)$ is disconnected, then $G$ is solvable.

Proof. Let $G$ be a counterexample with minimal order. Then, $G$ is non-solvable and $\Gamma_{r}^{*}(G)$ is disconnected. By Theorem 7, we get that $\Gamma_{r}^{*}(G)$ has exactly two connected components with vertex sets $\pi_{1}$ and $\pi_{2}$, respectively. We can assume that $G$ has no normal Sylow 2-subgroup, since, otherwise, by the Feit-Thompson theorem, $G$ is solvable. By Corollary 1, $G$ has a nontrivial real primary $2^{\prime}$-element $x$. Then, $\left|x^{G}\right|$ is even and hence $2 \in V_{r}^{*}(G)$. We assume that $2 \in \pi_{1}$. Therefore, all vertices in $\pi_{2}$ are odd primes. We divide the remaining proof into three parts.

(1) $G$ is not a non-abelian simple group.

By Lemma 11, there exists an involution $i \in G$ such that $\left|i^{G}\right|>1$ is a $\pi_{2}$-number and $\mathrm{C}_{G}(i)$ has a normal Sylow 2-subgroup, say $S$. As $\left|G: C_{G}(i)\right|$ is odd, $S$ is also a Sylow 2-subgroup of $G$. Notice that $C_{G}(i)$ is solvable. Now, for a real primary $2^{\prime}$-element $x \in G$, $\left|x^{G}\right|$ is even and so it is a $\pi_{1}$ number. Thus, $\left(\left|x^{G}\right|,\left|i^{G}\right|\right)=1$. It follows from ([21], Theorem 2) that $G$ is not a non-abelian simple group:

(2) $G=O^{2^{\prime}}(G)$ and $O_{2^{\prime}}(G)=1$.

Since $G$ is not 2-closed, by Theorem $8(1), \Gamma_{r}^{*}\left(O^{2^{\prime}}(G)\right)$ is disconnected. If $O^{2^{\prime}}(G)<G$, then $O^{2^{\prime}}(G)$ is solvable by the minimality of $|G|$. Therefore, $G$ is solvable since $G / O^{2^{\prime}}(G)$ is solvable, a contradiction. Thus, $G=O^{2^{\prime}}(G)$.

Since $G$ is not solvable, $G=O^{2^{\prime}}(G)$ is not 2-nilpotent. Hence, by Theorem 8(2), $\Gamma_{r}^{*}\left(G / O_{2^{\prime}}(G)\right)$ is disconnected. If $O_{2^{\prime}}(G)$ is nontrivial, then, by the minimality of $|G|$, we get $G / O_{2^{\prime}}(G)$ is solvable, and so is $G$. Thus, we must have $O_{2^{\prime}}(G)=1$.

(3) The final contradiction.

Let $M$ be a maximal normal subgroup of $G$. If $|G / M|_{2}=2$, then, by hypothesis, $|M|_{2}=2$ and $M$ must be solvable (see ([12], Theorem 1.35)), and so is $G$, a contradiction. The remaining cases where $|G / M|_{2}=4$ or $2 \nmid|G / M|$ both contradict (2).

\section{Conclusions}

By mainly considering the lengths of a portion of real classes, and using the prime graph $\Gamma_{r}^{*}(G)$ in particular, we obtain some information about the structure of $G$. We show that even this small subset of the set $\operatorname{cs}_{r}(G)$, with other proper conditions in occasional cases, still encodes a lot of structural information on $G$. This served as a background to continue the investigation of the influence of real elements and also the lengths of certain real classes on the structure of groups.

On the other hand, many authors have been inspired by looking at connections between characters and conjugacy classes, and, of course, between real characters and real classes (see [2,22-24]). Our works may shed some light on this research.

Author Contributions: Investigation, writing original draft: S.Y.; supervision, review and editing: X.L. Both authors have read and agreed to the published version of the manuscript.

Funding: This research was funded by the National Natural Science Foundation of China (Grant No. 11871360).

Institutional Review Board Statement: Not applicable.

Informed Consent Statement: Not applicable.

Data Availability Statement: Not applicable.

Conflicts of Interest: The authors declare no conflict of interest.

\section{References}

1. Alfandary, G. On graphs related to conjugacy classes of groups. Israel J. Math. 1994, 86, 211-220. [CrossRef]

2. Dolfi, S.; Navarro, G.; Tiep, P.H. Primes dividing the degrees of the real characters. Math. Zeit. 2008, 259, 755-774. [CrossRef]

3. Beltrán, A.; Felipe, M.J. Some class size conditions implying solvability of finite groups. J. Group Theory 2006, 9, 787-797. [CrossRef] 
4. Guralnick, R.M.; Navarro, G. Squaring a conjugacy class and cosets of normal subgroups. Proc. Amer. Math. Soc. 2016, 144, 1939-1945. [CrossRef]

5. Bianchi, M.; Brough, J.; Camina, R.D.; Pacifici, E. On vanishing class sizes in finite groups. J. Algebra 2017, 489, 446-459. [CrossRef]

6. Felipe, M.J.; Martínez-Pastor, A.; Ortiz-Sotomayor, V.M. Square-free class sizes in products of groups, J. Algebra 2017, 491, 190-206. [CrossRef]

7. Guralnick, R.M.; Navarro, G.; Tiep, P.H. Real class sizes and real character degrees. Math. Proc. Camb. Philos. Soc. 2011, 150, 47-71. [CrossRef]

8. Tong-Viet, H.P. Groups with some arithmetic conditions on real class sizes. Acta Math. Hungar. 2013, 140, 105-116. [CrossRef]

9. Tong-Viet, H.P. Real class sizes. Israel J. Math. 2018, 228, 753-769. [CrossRef]

10. Dolfi, S.; Pacifici, E.; Sanus, L. Finite groups with real conjugacy classes of prime size. Israel J. Math. 2010, 175, 179-189. [CrossRef]

11. Guo, X.; Zhao, X.; Shum, K.P. On $p$-regular G-conjugacy classes and the $p$-structure of normal subgroups. Comm. Algebra 2009, 37, 2052-2059. [CrossRef]

12. Isaacs, I.M. Finite Group Theory; Graduate Studies in Mathematics; AMS: Providence, RI, USA, 2008 ; Volume 92.

13. Navarro, G.; Sanus, L.; Tong-Viet, H.P. Real characters and degrees. Israel J. Math. 2009, 171, 157-173. [CrossRef]

14. Chen, R.; Zhao, X. A criterion for a group to have nilpotent $p$-complements. Monatsh. Math. 2016, 179, 221-225. [CrossRef]

15. Beidleman, J.C.; Robinson, D.J.S. On finite groups satisfying the permutizer condition. J. Algebra 1997, 191, 686-703. [CrossRef]

16. Griess, R.L.; Finite groups whose involutions lie in the center. Quart. J. Math. 1978, 29, 241-247. [CrossRef]

17. Conway, J.H.; Curtis, R.T.; Norton, S.P.; Parper, R.A.; Wilson, R.A. Atlas of Finite Groups; Oxford University Press: Eynsham, UK, 1985.

18. Gorentein, D. Finite Groups, 2nd ed.; Chelsea Publishing Company: New York, NY, USA, 1980.

19. Isaacs, I.M.; Navarro, G. Normal p-complements and fixed elements. Arch. Math. 2010, 95, 207-211. [CrossRef]

20. Lu, Z.; Zhang, J. On the diameter of a graph related to $p$-regular conjugacy classes of finite groups. J. Algebra 2000, 231, 705-712. [CrossRef]

21. Fisman, E.; Arad, Z. A proof of Szep's conjecture on nonsimplicity of certain finite groups. J. Algebra 1987, 108, 340-354. [CrossRef]

22. Liu, X. On lengths of conjugacy classes and character degrees in finite groups. Comm. Algebra 2006, 34, 1443-1449. [CrossRef]

23. Dan, R. Finite groups with three rational conjugacy classes. Arch. Math. 2007, 110, 1-10.

24. Robati, S.M. Real-imaginary conjugacy classes and real-imaginary irreducible characters in finite groups. Math. Notes 2018, 103, 251-258. [CrossRef] 